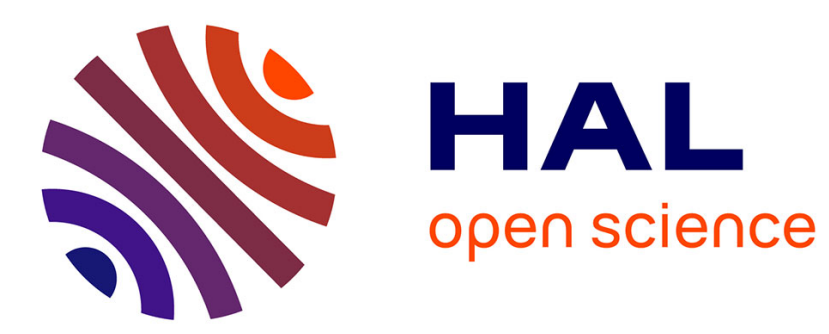

\title{
Multichannel Electrocardiogram Decomposition Using Periodic Component Analysis
}

\author{
Reza Sameni, Christian Jutten, Mohammad B. Shamsollahi
}

\section{To cite this version:}

Reza Sameni, Christian Jutten, Mohammad B. Shamsollahi. Multichannel Electrocardiogram Decomposition Using Periodic Component Analysis. IEEE Transactions on Biomedical Engineering, 2008, 55 (8), pp.1935-1940. 10.1109/TBME.2008.919714 . hal-00306449

\section{HAL Id: hal-00306449 \\ https://hal.science/hal-00306449}

Submitted on 25 Jul 2008

HAL is a multi-disciplinary open access archive for the deposit and dissemination of scientific research documents, whether they are published or not. The documents may come from teaching and research institutions in France or abroad, or from public or private research centers.
L'archive ouverte pluridisciplinaire HAL, est destinée au dépôt et à la diffusion de documents scientifiques de niveau recherche, publiés ou non, émanant des établissements d'enseignement et de recherche français ou étrangers, des laboratoires publics ou privés. 


\title{
Multichannel Electrocardiogram Decomposition Using Periodic Component Analysis
}

\author{
Reza Sameni*, Christian Jutten, and Mohammad B. Shamsollahi
}

\begin{abstract}
In this letter, we propose the application of the generalized eigenvalue decomposition for the decomposition of multichannel electrocardiogram (ECG) recordings. The proposed method uses a modified version of a previously presented measure of periodicity and a phase-wrapping of the RR-interval, for extracting the "most periodic" linear mixtures of a recorded dataset. It is shown that the method is an improved extension of conventional source separation techniques, specifically customized for ECG signals. The method is therefore of special interest for the decomposition and compression of multichannel ECG, and for the removal of maternal ECG artifacts from fetal ECG recordings.
\end{abstract}

Index Terms-Blind source separation, fetal ECG extraction, generalized eigenvalue decomposition.

\section{INTRODUCTION}

I NDEPENDENT component analysis (ICA) has been extensively used for solving blind source separation (BSS) problems and extracting independent components embedded in multichannel recordings. ICA techniques are usually based on the maximization of some nonlinear criterion as a measure of component independence. However, for pseudoperiodic signals such as the electrocardiogram (ECG), the temporal structure of the signal is rich in information. For such signals, a measure of periodicity of the extracted signals, both clinically and mathematically, is a more appropriate criterion than independence. In some recent works, the temporal periodicity of ECG signals has been exploited in source separation algorithms [1]. These methods are, however, of the same order of complexity as conventional ICA algorithms, and are basically designed for signals having a constant fundamental period; an assumption that is not true for real ECG recordings.

In this letter, we present an extension of previous source separation methods that is specifically customized for the ECG. The proposed method is partially based on the notion of periodic component analysis ( $\pi \mathrm{CA}$ ) proposed in [2] and generalized eigenvalue decomposition [3]. The relationships between this

Manuscript received September 7, 2007; revised November 15, 2007. Asterisk indicates corresponding author.

*R. Sameni is with the Biomedical Signal and Image Processing Laboratory (BISIPL), School of Electrical Engineering, Sharif University of Technology, 11365-9313 Tehran, Iran. He is also with the Laboratory of Grenoble Image Parole Signal Automatique (GIPSA-LAB), INPG, 38031 Grenoble, France (e-mail: reza.sameni@gmail.com).

C. Jutten is with the Laboratory of Grenoble Image Parole Signal Automatique (GIPSA-LAB), INPG, 38031 Grenoble, France (e-mail: christian.jutten @ inpg.fr).

M. B. Shamsollahi is with the Biomedical Signal and Image Processing Laboratory (BiSIPL), School of Electrical Engineering, Sharif University of Technology, 11365-9313 Tehran, Iran (e-mail: mbshams@ sharif.ir).

Color versions of one or more of the figures in this paper are available online at http://ieeexplore.ieee.org.

Digital Object Identifier 10.1109/TBME.2008.919714

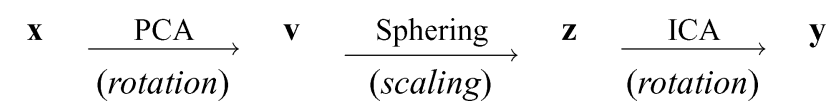

Fig. 1. General scheme of ICA algorithms with spatial whitening.

method and well-known ICA techniques such as AMUSE [4], JADE [5], and SOBI [6] are also discussed. It will be shown that for ECG signals, the method has several benefits over conventional ICA and is applicable to both adult and fetal ECG embedded in noise.

\section{BACKGROUND}

\section{A. Generalized Eigenvalue Decomposition}

For $n \times n$ symmetric matrices $A$ and $B$, the problem of generalized eigenvalue decomposition (GEVD) [7] of the matrix pair $(A, B)$ consists of finding the matrices $U$ and $D$, such that:

$$
U^{T} A U=D \quad, \quad U^{T} B U=I
$$

where $D$ is the diagonal generalized eigenvalue matrix corresponding to the eigenmatrix $U$, with real eigenvalues sorted in ascending order on its diagonal. ${ }^{1}$ As seen from (1), $U$ is a transformation that simultaneously diagonalizes $A$ and $B$.

\section{B. ICA Versus Generalized Eigenvalue Decomposition}

In the context of ICA, we practically have a finite number of samples of an $n$-dimensional observation vector $\mathbf{x}(t)=$ $\left[x_{1}(t), \ldots, x_{n}(t)\right]^{T}$, and seek for a linear mixture of these observations that maximizes some measure of independence, or namely a contrast function. Under some general assumptions, the estimated components are solutions of a BSS problem with a linear latent variable model. Moreover, most ICA algorithms perform spatial whitening on the dataset, which, as shown in Fig. 1, only leaves a rotation matrix to be estimated by maximizing the contrast function of ICA.

An algebraic approach to ICA is to diagonalize a set of matrices containing second or higher-order statistics of the dataset [5]. For signals with temporal structure, there are various algorithms that use this algebraic approach. For example, for a wide-sense stationary or cyclostationary real observation vector $\mathbf{x}(t)$, if we define the covariance matrix as:

$$
C_{x}(\tau)=E_{t}\left\{\mathbf{x}(t+\tau) \mathbf{x}(t)^{T}\right\}
$$

where $E_{t}\{\cdot\}$ indicates averaging over $t$, the AMUSE algorithm is a method that jointly whitens the data and diagonalizes $C_{x}(\tau)$

\footnotetext{
${ }^{1}$ In the problem of interest, $A$ and $B$ are symmetric positive-definite matrices; therefore, the eigenvalues of $D$ are real and positive [7].
} 
for some arbitrary $\tau$, i.e., the solution of the GEVD problem of the matrix pair $\left(C_{x}(\tau), C_{x}(0)\right)$ [3]. However, it is known that no more than two matrices may be exactly diagonalized by a quadratic transformation of the form (1), except when they belong to the same eigenspace [6]. Due to this fact, methods have been proposed that approximately diagonalize a set of desired matrices, and are more robust to data outliers and computational errors, as compared with AMUSE [8]. In this context, the SOBI algorithm is an example of a time-domain algorithm that whitens the data and approximately diagonalizes $C_{x}(\tau)$ for several time-lags $\tau$ [6]. Similar time-domain methods have also been proposed for cyclostationary sources, in which the data is again prewhitened and matrices representing cyclostationary statistics of the dataset are (approximately) diagonalized [9]. However, the final output of any of these approximate diagonalization methods is a linear transformation of the form:

$$
\begin{aligned}
\mathbf{y}(t) & =U^{T} \mathbf{x}(t) \\
U & =Q \Lambda^{-1 / 2} R
\end{aligned}
$$

where $Q$ and $\Lambda$ are, respectively, the eigenmatrix and eigenvalue matrix of $C_{x}(0)^{2}$, and $R$ is the rotation matrix found by maximizing the contrast function of the ICA method. In (3), the terms $Q, \Lambda^{-1 / 2}$, and $R$, respectively, correspond with the principal component analysis (PCA), sphering, and ICA steps indicated in Fig. 1. It is easy to show that the transformation $U$ diagonalizes the following symmetric matrix:

$$
\Gamma=Q \Lambda^{1 / 2} R D R^{T} \Lambda^{1 / 2} Q^{T}
$$

where $D$ is an arbitrary diagonal matrix. ${ }^{3}$ It therefore follows that the matrix pair $\left(\Gamma, C_{x}(0)\right)$ satisfies (1).

Equation (4) implies that for prewhitened data and for a rotation matrix $R$ found by maximizing any ICA contrast function, there exists a set of matrices $\Gamma$ that are exactly diagonalizable via GEVD. The matrix $\Gamma$, by itself is not necessarily any of the second or higher-order statistics matrices of the data; but contains some overall statistics of the data that is exactly diagonalized through the linear transformation $U$.

From this point of view, any ICA algorithm of the form of Fig. 1 may be transformed into a GEVD problem for diagonalizing a single matrix containing some cross-statistical measure of the multichannel dataset. This suggests that, in some applications, instead of forming a set of matrices containing conventional statistics of the data, such as the second or higher-order statistics, and approximately diagonalizing these matrices, we can directly use the a priori information about the desired signals (e.g., their periodicity) to form an ad hoc symmetric matrix $\Gamma$, and seek for linear transformations that diagonalize this matrix, i.e., decorrelate the ad hoc statistics. The matrix $\Gamma$ may, for example, be a covariance-like matrix, as defined in (2), but using a time-varying time lag $\tau$ that is derived from our prior knowledge of the dataset. This idea is investigated for ECG signals in the following sections.

\footnotetext{
${ }^{2} C_{x}(0)=Q \Lambda Q^{T}$

${ }^{3}$ In fact, in (4), the term $R D R^{T}$ represents an arbitrary matrix from the eigenspace of $R$ and $\Gamma=U^{-T} D U^{-1}$.
}

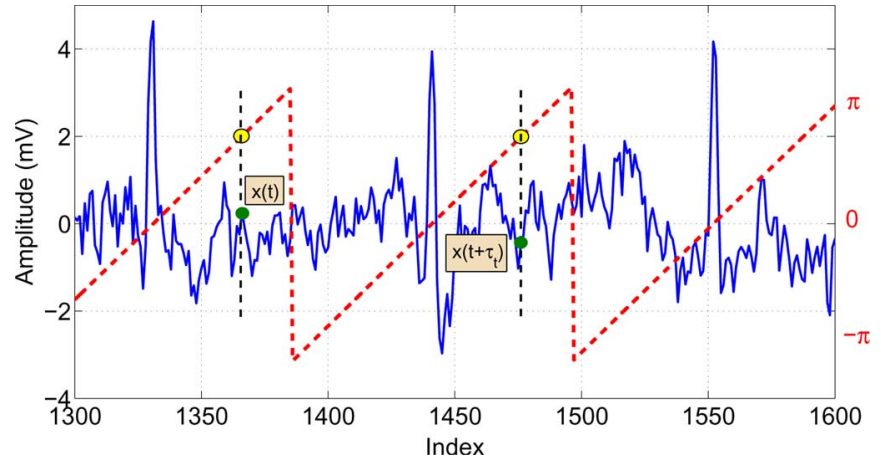

Fig. 2. Illustration of the phase assignment procedure used for calculating $\tau_{t}$ in each ECG beat.

\section{Periodic Component Analysis}

Here, we restate the problem of periodic component analysis, adapted from [2], which is merely a restatement of the AMUSE algorithm derived from a measure of periodicity.

Given an $n$-dimensional observation vector $\mathbf{x}(t)=$ $\left[x_{1}(t), \ldots, x_{n}(t)\right]^{T}$, we seek for the linear mixture $s(t)=$ $\mathbf{w}^{T} \mathbf{x}(t)$ with a maximal periodic structure that minimizes the following measure of periodicity:

$$
\epsilon(\mathbf{w}, \tau)=\frac{\sum_{t}|s(t+\tau)-s(t)|^{2}}{\sum_{t}|s(t)|^{2}}
$$

where $\mathbf{w}=\left[w_{1}, \ldots, w_{n}\right]^{T}$ and $\tau$ is the period of interest. It can be shown that (5) may be rearranged as follows:

$$
\epsilon(\mathbf{w}, \tau)=\frac{\mathbf{w}^{T} A_{x}(\tau) \mathbf{w}}{\mathbf{w}^{T} C_{x}(0) \mathbf{w}}=2\left[1-\frac{\mathbf{w}^{T} C_{x}(\tau) \mathbf{w}}{\mathbf{w}^{T} C_{x}(0) \mathbf{w}}\right]
$$

where

$$
\begin{aligned}
A_{x}(\tau) & =E_{t}\left\{[\mathbf{x}(t+\tau)-\mathbf{x}(t)][\mathbf{x}(t+\tau)-\mathbf{x}(t)]^{T}\right\} \\
& =2 C_{x}(0)-2 C_{x}(\tau) .
\end{aligned}
$$

In the second part of (7), we have used the symmetry of the matrix $C_{x}(\tau)$. Then, using the Rayleigh-Ritz theorem from linear algebra [7], it follows that the weight vector $\mathbf{w}$ minimizing (6) is given by the eigenvector corresponding to the smallest generalized eigenvalue of the matrix pair $\left(A_{x}(\tau), C_{x}(0)\right)$, or equivalently, the largest generalized eigenvalue of $\left(C_{x}(\tau), C_{x}(0)\right)$. As with (1), by assuming $D$ as the diagonal generalized eigenvalue matrix corresponding to the eigenmatrix $U$, with real eigenvalues sorted in descending order on its diagonal, the transformation $U^{T} \mathbf{x}(t)$ gives the most periodic components in descending order of periodicity.

\section{METHOD}

ECG signals have a pseudoperiodic structure that is repeated in every cycle of the ECG beat. However, normal ECGs can have RR-period deviations of up to $20 \%$ (c.f. [10]), which means that a constant period $\tau$, as defined in the previous section, does not fully describe the periodicity of the ECG.

For such signals, we propose to use a time-varying period that is updated on a beat-to-beat basis. For this, as shown in Fig. 2, 


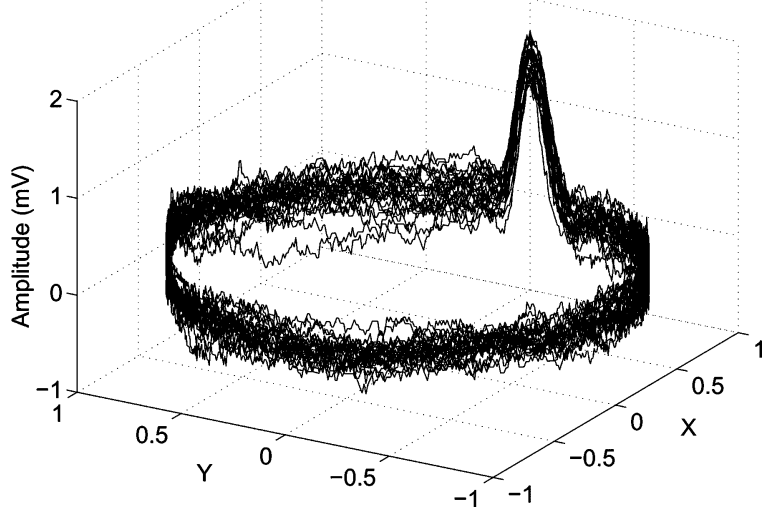

Fig. 3. Polar representation of a noisy ECG using the ECG phase $\phi(t)$.

by detecting the R-peaks of the ECG, a linear phase $\phi(t)$ ranging from $-\pi$ to $\pi$ is assigned to each ECG sample, with the R-peak being fixed at $\phi(t)=0$. The linear phase $\phi(t)$, provides a means for phase-wrapping the RR-interval onto the $[-\pi, \pi]$ interval. Therefore, the ECG - regardless of its RR-interval deviationsmay be converted to a polar representation in which the ECG components in different beats, such as the P, Q, R, S, and Twaves, are more or less phase-aligned with each other, especially over the QRS segment (Fig. 3).

On the other hand, minimizing (6), requires the calculation of the cross-correlation between the samples having a time-lag $\tau$ in different channels. So, in order to apply $\pi \mathrm{CA}$ to ECG signals, we can replace the constant time-lag $\tau$ in (7), with a variable $\tau_{t}$ that is calculated from $\phi(t)$ from beat-to-beat. Therefore, in each ECG cycle, the sample at the time-instant $t$ is compared with the sample $t+\tau_{t}$, namely its dual sample, which is the sample with the same phase value in the successive ECG beat. The timevarying period $\tau_{t}$ may be mathematically defined as follows:

$$
\tau_{t}=\min \{\tau \mid \phi(t+\tau)=\phi(t), \tau>0\} .
$$

Using this definition, the covariance matrix defined in (2) is redefined as follows:

$$
\tilde{C}_{x}=E_{t}\left\{\mathbf{x}\left(t+\tau_{t}\right) \mathbf{x}(t)^{T}\right\} .
$$

Moreover, in order to assure the symmetry of $\tilde{C}_{x}$ and the realness of its eigenvalues, the following step is required in practice:

$$
\tilde{C}_{x}=\left(\tilde{C}_{x}+\tilde{C}_{x}^{T}\right) / 2 .
$$

In fact, if we consider the polar representations of each of the channels of $\mathbf{x}(t)$ (as in Fig. 3), the matrix $\tilde{C}_{x}$ represents the covariance of the polar representation around its mean value. Following the discussions in Section II-B, $\tilde{C}_{x}$ is the matrix containing the ad hoc statistics of the ECG, i.e., a measure of ECG periodicity extracted from the ECG R-peak information.

Next we find $U$, the GEVD solution of the $\left(\tilde{C}_{x}, C_{x}(0)\right)$ pair with the eigenvectors ranked in descending order of their corresponding generalized eigenvalues. The desired signal vector $\mathbf{y}(t)=\left[y_{1}(t), \ldots, y_{n}(t)\right]^{T}$ is then found from (3). The components of $\mathbf{y}(t)$ are sorted according to the amount of their periodicity, relative to the heart beat. In other words, $y_{1}(t)$ is the

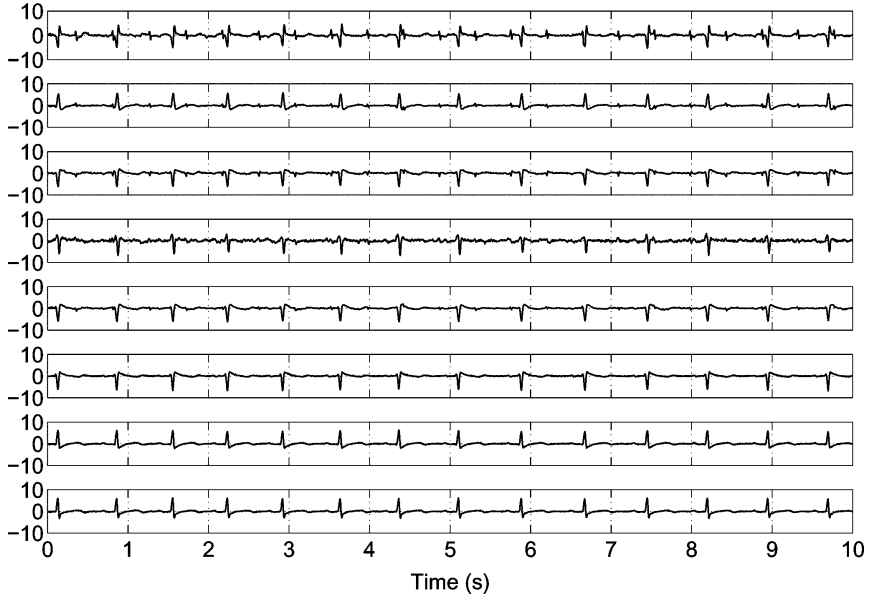

Fig. 4. DaISy dataset consisting of five maternal abdominal and three thoracic channels [11].

most periodic component and $y_{n}(t)$ is the least periodic, with respect to the R-peaks of the ECG.

The proposed method is rather flexible, and may be extended to other ad hoc statistics extracted from ECG recordings. For instance, for the problem of fetal ECG extraction, if we define $\phi_{m}(t)$ and $\phi_{f}(t)$ as the maternal and fetal ECG phases found from the maternal and fetal R-peaks, $\tilde{C}_{x}^{m}$ and $\tilde{C}_{x}^{f}$ representing the covariance matrices of the mother and fetus, are found by averaging (9) over the maternal and fetal periods, respectively. Then, the matrix $\tilde{C}_{x}$ used in the GEVD may be set to any of the following matrices:

$$
\begin{aligned}
\tilde{C}_{x} & =\tilde{C}_{x}^{m} \\
\tilde{C}_{x} & =\tilde{C}_{x}^{f} \\
\tilde{C}_{x} & =\tilde{C}_{x}^{m}-\tilde{C}_{x}^{f} .
\end{aligned}
$$

If we assume the data to be prewhitened, the diagonalization of the matrices defined in (11) is, respectively, equivalent to finding 1) the most periodic components with respect to the maternal ECG, 2) the most periodic components with respect to the fetal ECG, and 3) the most periodic components with respect to the maternal ECG while being the least periodic components with respect to the fetal ECG. In this latter case, the extracted components should gradually change from the maternal ECG to the fetal ECG, from the first to the last component. ${ }^{4}$ It should, of course, be noted that the last two cases are difficult to implement in practice, as they require the prior extraction of the fetal R-peaks to form the $\tilde{C}_{x}^{f}$ matrix.

\section{RESUlTS}

The well-known DaISy fetal ECG database is used for illustration [11]. The database consists of five abdominal and three thoracic channels recorded from the abdomen and chest of a pregnant woman with a sampling rate of $250 \mathrm{~Hz}$. The eight channels of the dataset may be seen in Fig. 4.

\footnotetext{
${ }^{4}$ Note that, in the last definition of (11), $\tilde{C}_{x}$ is not necessarily positive definite However, due to its symmetry, the generalized eigenvalues are real and may be ranked in ascending/descending order.
} 


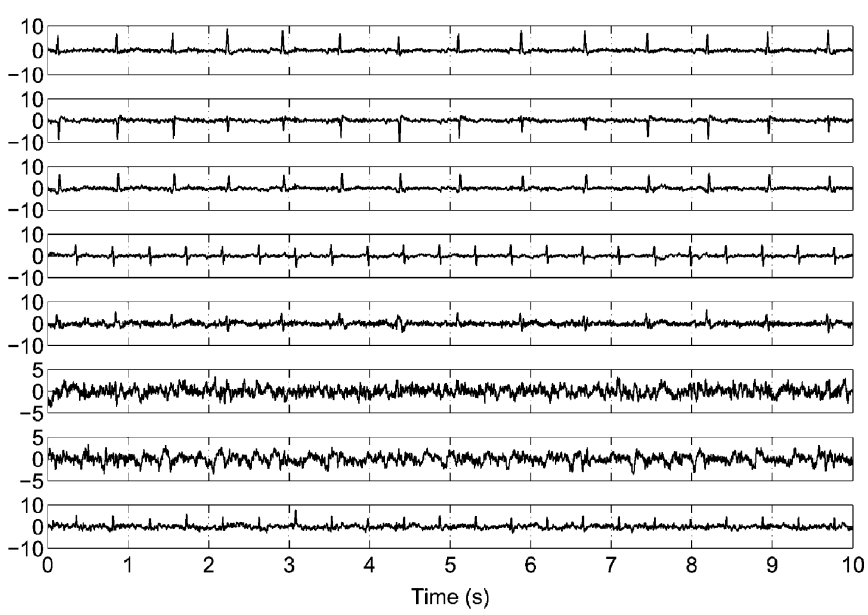

Fig. 5. Independent components extracted from the dataset of Fig. 4, using JADE. Notice that components $1,2,3$, and 5 correspond to the maternal subspace and components 4 and 8 to the fetal subspace.

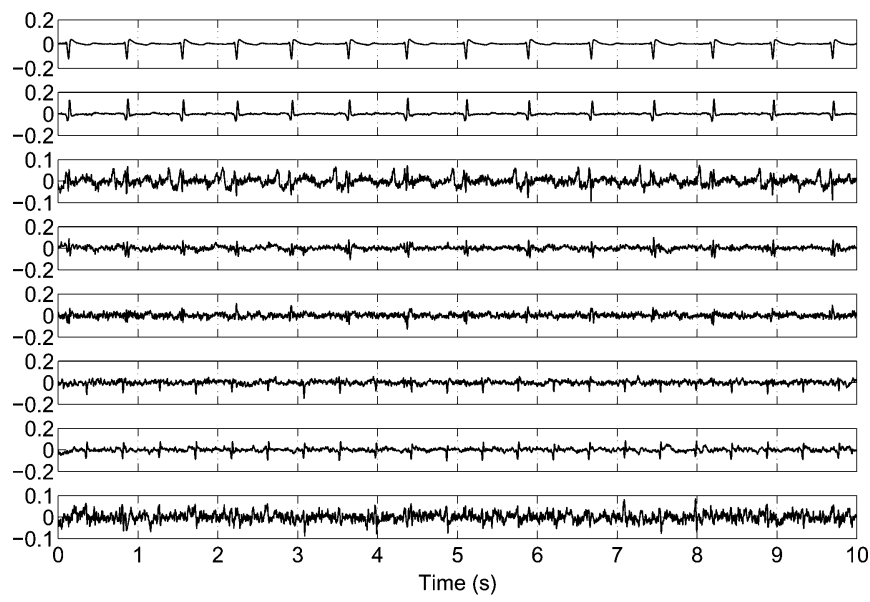

Fig. 6. Periodic components extracted from the dataset with maternal ECG beat synchronization. The maternal ECG contribution reduces from top to bottom.

The JADE algorithm [5], is used as the benchmark ICA method. The eight independent components extracted by this algorithm are depicted in Fig. 5.

By performing R-wave detection on one of the maternal thoracic channels, the maternal ECG phase $\phi_{m}(t)$ is calculated according to the explanations of the previous section. Next, the time-varying maternal ECG period $\tau_{t}^{m}$ is calculated, from which the matrix $\tilde{C}_{x}$ and the generalized eigenmatrix $U$ of the $\left(\tilde{C}_{x}, C_{x}(0)\right)$ pair are found and sorted in descending order of the eigenvalues. The resultant periodic components calculated from (3) are depicted in Fig. 6. As seen in Fig. 6, the first component (corresponding to the largest eigenvalue) has the most resemblance with the maternal ECG, while as the eigenvalues decrease, the signals become less similar to the maternal ECG. Interestingly, two of the extracted components (components six and seven) are the fetal components. This can be explained by considering that $\pi \mathrm{CA}$ is ranking the extracted components according to their resemblance with the maternal ECG period, while the fetal components do not resemble the maternal ECG,

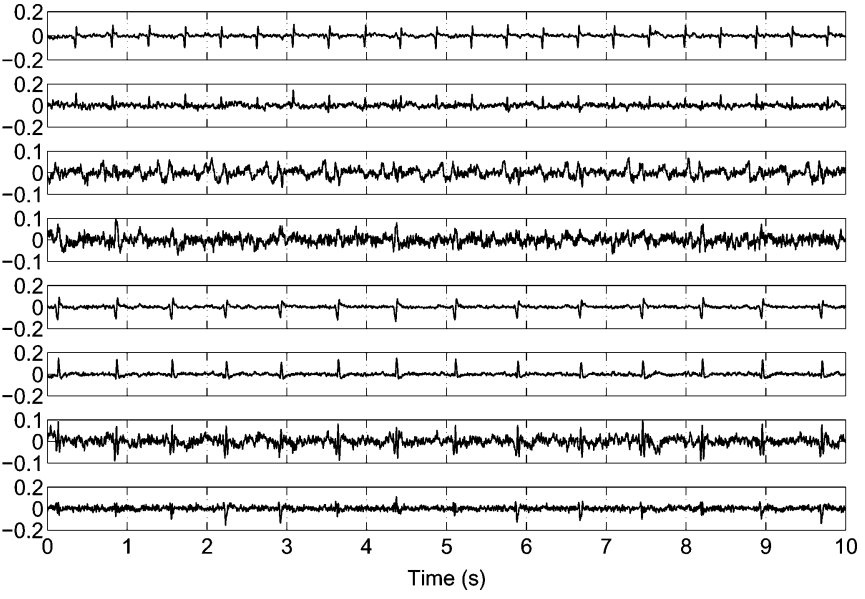

Fig. 7. Periodic components extracted from the dataset with fetal ECG beat synchronization. Notice that the fetal ECG contribution reduces from top to bottom.

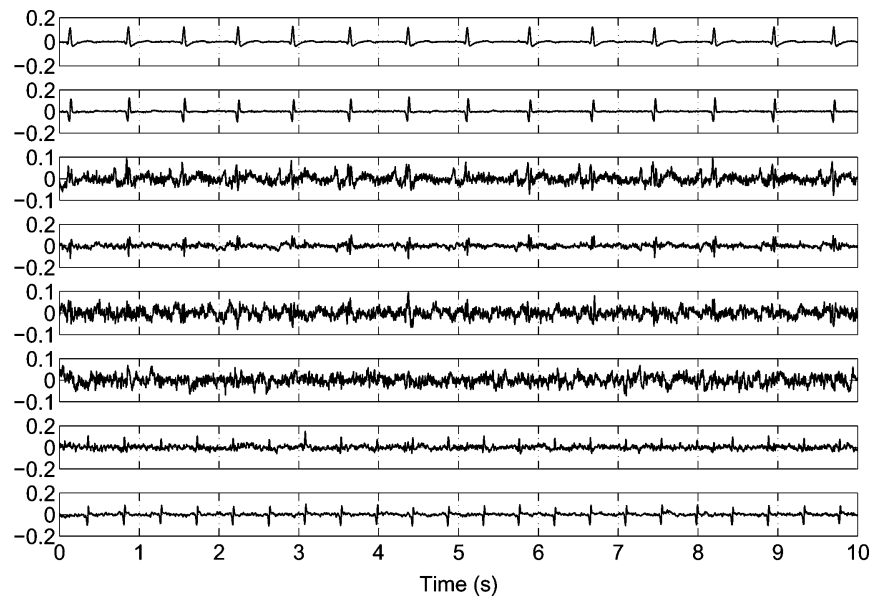

Fig. 8. Periodic components extracted from the dataset with maternal \& fetal ECG beat synchronization. The maternal (fetal) ECG contribution reduces (increases) from top to bottom.

when averaged synchronously with respect to the maternal Rpeaks. The fetal components are therefore extracted among the last components.

As explained in Section III, it is also possible to consider the fetal ECG periodicity in the matrix $\tilde{C}_{x}$, which requires the fetal R-peaks for extracting the time-varying fetal period $\tau_{t}^{f}$. For this, the fetal ECG component extracted by JADE in the fourth channel of Fig. 5 is used for fetal R-peak detection and phase calculation. Having calculated the fetal ECG phase $\phi_{f}(t)$, the previously explained procedures are repeated to extract the periodic components of the fetal ECG. The resultant periodic components are depicted in Fig. 7. This time we see that the extracted components are ranked according to their resemblance with the fetal ECG.

The last results correspond to the last type of covariance matrix defined in $(11 \mathrm{c})$. The covariance matrix $\tilde{C}_{x}$ for this part is calculated from the difference of $\tilde{C}_{x}^{m}$ and $\tilde{C}_{x}^{f}$. After performing the previously explained GEVD stages, the periodic components are found from (3). These components are depicted in Fig. 8. 
As expected before, the first component has the most resemblance with the maternal ECG, while the last component mostly resembles the fetal ECG and the intermediate components are mixtures of maternal and fetal components and noise.

\section{DISCUSSION AND CONCLUSION}

The proposed method is able to extract the "most periodic" components corresponding to a desired ECG signal from a set of multichannel recordings by forming some covariance-like matrix and jointly diagonalizing this matrix and the actual covariance matrix of the dataset. The intuition behind this method is to find any periodic structure that is synchronous with the reference ECG R-peaks extracted from a suitably clean ECG reference. As was explained in Section II-B, the bases of the proposed method are rather similar to PCA and ICA. However, due to the proper use of the temporal pseudoperiodicity of the ECG, the method has some interesting benefits over conventional source separation techniques, which are noted in the following:

1) From the physiological point of view, the independence criterion of conventional ICA has been replaced with a periodic temporal structure criterion, which is a more reasonable assumption for ECG signals and in accordance with the clinical intuition about the ECG. In fact, cardiologists are not familiar with the interpretation of independent components extracted from multichannel recordings, but they are interested in periodic structures that are repeated in each ECG beat.

2) From the mathematical point of view, all the temporal information of the ECG is gathered in the matrix $\tilde{C}_{x}$. Therefore, the conventional iterative ICA algorithm is replaced by a closed-form solution consisting of an initial $\mathrm{R}$-wave detection step, covariance matrix calculation, and a single step of GEVD. The method is therefore more time-efficient.

3) The extracted components are ranked according to their degree of synchronization (periodicity) with the R-peaks, while in conventional ICA, it is not possible to predict the order of the extracted components. This feature is very helpful, especially for automating the removal of the maternal ECG from fetal ECG recordings, or generally for removing cardiac interference from multichannel biosignals.

4) Due to the mean-square-error compression property of the eigenvalue decomposition ( [13], Ch. 6), the ECG signal is concentrated within the least number of components. In other words, by using the proposed method, we achieve a minimal representation of the ECG signal. This feature can be used in ECG compression, as we can define some threshold on the extracted eigenvalues and remove the components corresponding to the smaller eigenvalues by simple thresholding or through a hypothesis test. In this way, since the components are ranked according to their resemblance with the ECG, we are sure that the "least important" components have been removed. This is, however, not the case for ICA or PCA compression techniques when applied to the ECG, since the low variance components extracted by these methods can convey important parts of the ECG morphology and may not be removed by thresholding [14].

5) The eigenvalues found from (1) are also a measure of the noisiness of the extracted component and may be used for thresholding the minor components. In the general BSS problem, if we consider the observed data to follow the latent variable model $\mathbf{x}=H \mathbf{s}+\mathbf{n}$, any linear combination of the data $\mathbf{x}$ will contain some noise [15]. However depending on the criterion that is used, the noise may be accumulated in a few undesired components while keeping the desired components such as the ECG cleaner than the rest of the components. In fact, if we consider any source of aperiodicity as noise, the proposed method may be interpreted as a transformation that is distributing the noise variance in the less important components. This is an important issue that is not achieved with conventional ICA, as they seek for the most independent components and not the most periodic (least noisy) ones.

6) The solutions of GEVD problems are generally more susceptible to noise, as compared with joint approximate diagonalization methods [6]. However, in the proposed method, although only two matrices are jointly diagonalized, the results are still robust to deviations of heartbeat and noise. The reason is that the time lags $\tau_{t}$ required in the calculation of $\tilde{C}_{x}$, are extracted from the beat-to-beat information of the ECG. The robustness may, however, be improved in future works, if we split the overall information of the RR-interval that is carried by $\tilde{C}_{x}$, into several matrices that contain local information of the ECG cycle, such as the P, QRS, and T-segments. These matrices can then be approximately diagonalized, using joint approximate diagonalization methods [8].

In future works, the performance of the proposed method should also be studied for real and simulated multichannel ECG signals [16], with different degrees of morphological and heartbeat deviations, and in different sampling rates and signal-to-noise ratios.

\section{REFERENCES}

[1] M. G. Jafari, W. Wang, J. A. Chambers, T. Hoya, and A. Cichocki, "Sequential blind source separation based exclusively on second-order statistics developed for a class of periodic signals," IEEE Trans. Signal Process., vol. 54, no. 3, pp. 1028-1040, Mar. 2006.

[2] L. K. Saul and J. B. Allen, "Periodic component analysis: An eigenvalue method for representing periodic structure in speech.," in NIPS, [Online]. 2000, pp. 807-813. Available: http://www.cs.cmu.edu/Groups/ NIPS/00papers-pub-on-web/SaulAllen.pdf

[3] L. Parra and P. Sajda, "Blind source separation via generalized eigenvalue decomposition," J. Mach. Learn. Res., vol. 4, pp. 1261-1269, 2003.

[4] L. Tong, R.-W. Liu, V. Soon, and Y.-F. Huang, "Indeterminacy and identifiability of blind identification," IEEE Trans. Circuits Syst., vol. 38, no. 5, pp. 499-509, May 1991.

[5] J.-F. Cardoso and A. Souloumiac, "Blind beamforming for non Gaussian signals," Inst. Elect. Eng. Proc. F Radar Signal Process., vol. 140, no. 6 , pp. 362-370, Dec. 1993.

[6] A. Belouchrani, K. Abed-Meraim, J.-F. Cardoso, and E. Moulines, "A blind source separation technique using second-order statistics," IEEE Trans. Signal Process., vol. 45, no. 2, pp. 434-444, Feb. 1997.

[7] G. Strang, Linear Algebra and Its Applications, 3rd ed. Pacific Grove, CA: Brooks/Cole, 1988. 
[8] J.-F. Cardoso, "High-order contrasts for independent component analysis," Neural Comput., vol. 11, no. 1, pp. 157-192, 1999.

[9] A. Ferreol and P. Chevalier, "On the behavior of current second and higher order blind source separation methods for cyclostationary sources," IEEE Trans. Signal Process, vol. 48, no. 6, pp. 1712-1725, Jun. 2000.

[10] The MIT-BIH Normal Sinus Rhythm Database. [Online]. http://www. physionet.org/physiobank/database/nsrdb/.

[11] B. De Moor, Database for the Identification of Systems (DaISy), [Online]. Available at: http://homes.esat.kuleuven.be/ $/$ smc/daisy/

[12] J.-F. Cardoso, "Multidimensional independent component analysis," in Proc. IEEE Int. Conf. Acoust. Speech Signal Process. (ICASSP 1998) vol. 4, pp. 1941-1944.

[13] A. Hyvarinen, J. Karhunen, and E. Oja, Independent Component Analysis. New York: Wiley-Interscience, 2001

[14] R. Sameni, C. Jutten, and M. B. Shamsollahi, "What ICA provides for ECG processing: Application to noninvasive fetal ECG extraction," in Proc. Int. Symp. Signal Process. Inf. Technol. (ISSPIT 2006), Vancouver, Canada, pp. 656-661.

[15] M. Davies, "Identifiability issues in noisy ICA," IEEE Signal Process. Lett., vol. 11, no. 5, pp. 470-473, May 2004.

[16] R. Sameni, G. D. Clifford, C. Jutten, and M. B. Shamsollahi, "Multichannel ECG and noise modeling: Application to maternal and fetal ECG signals," EURASIP J. Adv. Signal Process., vol. 2007, 2007. ISSN 1687-6172, doi:10.1155/2007/43407. [Online]. Available: http:// www.hindawi.com/ GetArticle.aspx?doi=10.1155/2007/43407
Reza Sameni, photograph and biography not available at the time of publication.

Christian Jutten, photograph and biography not available at the time of publication.

Mohammad B. Shamsollahi, photograph and biography not available at the time of publication. 